\title{
Descripción del índice de oxigenación ajustado y del puntaje APPSen Ios pacientes adultos con SDRA de la Unidad de Cuidado Intensivo del Hospital Universitario Fundación Santa Fe de Bogotá.
}

Claudia M. Ramirez"12, Laura M. Castillo', Claudia P. Correa', Leidy P. Prada', Mónica P. Caicedo"13
spital Universitario Fundación Santa Fe de Bogotá, ${ }^{11}$ Universidad del Rosario, ${ }^{3}$ Universidad de los Andes. Santa Fe de Bogota

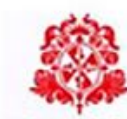

Universidad del Rosario

'Hospital Universitario Fundación Santa Fe de Bogotá, ${ }^{2}$ Universidad del Rosario, ${ }^{3}$ Universidad de los Andes.

\section{Introducción}

La predicción de desenlaces representa uno de los pilares en la práctica clínica habitual. En los pacientes con síndrome de dificultad respiratoria del adulto (SDRA), se requieren estrategias prácticas para la predicción de mortalidad.

\section{Objetivos}

Describir la severidad del compromiso por SDRA según el índice de oxigenación ajustado y el puntaje APPS en los pacientes adultos hospitalizados en la Unidad de Cuidado Intensivo en un hospital de IV nivel entre 2009-2018.

\section{Materiales y métodos}

Estudio corte transversal donde se recopiló la información de pacientes mayores de 18 años con diagnóstico de SDRA, con datos completos respecto a parámetros ventilatorios y gases arteriales. Análisis estadístico software STATA 11.

\section{Resultados}

En la tabla 1, 2 y 3 se muestran las características clínicas, ventilatorias y gasométricas de los pacientes incluidos en el análisis. La severidad del SDRA de acuerdo con la clasificación de Berlin se presenta en la tabla 4.

\begin{tabular}{|lc|}
\hline Tabla 1. Características de los pacientes. & \\
\hline Característica & $n=18$ \\
\hline Hombres, no. (\%) & $10(55.56)$ \\
\hline Edad en años, promedio (DE) & $64.55(13.44)$ \\
\hline Puntaje Apache II, promedio (DE) & $23.44(5.52)$ \\
\hline Puntaje SOFA, promedio (DE) & $9.94(4.15)$ \\
\hline Manejo en UCI & \\
\hline ECMO, no. (\%) & $2(11.11)$ \\
\hline Ventilación prono, no. (\%) & $5(27.78)$ \\
\hline Relajación neuromuscular, no. (\%) & $11(61.11)$ \\
\hline Desenlaces & \\
\hline Días hospitalización, mediana (Q1, Q3) & $21(15,32)$ \\
\hline Días UCl, mediana (Q1, Q3) & $14(8,19)$ \\
\hline Días ventilación, mediana (Q1, Q3) & $12.5(5,17)$ \\
\hline Mortalidad intrahospitalaria, no. (\%) & $11(61.11)$ \\
\hline
\end{tabular}

El promedio del índice de oxigenación ajustado fue 90.45 y el puntaje APPS fue de 5.94. Estos se asocian a una mortalidad aproximada del $50 \%$ a 28 días y $40.9 \%$ a 60 días, respectivamente.

\section{Tabla 2. Variables ventilatorias.}

\section{\begin{tabular}{|l}
\hline V \\
\hline Fi \\
\hline V \\
\hline Fr \\
\hline P \\
\hline P \\
\hline D \\
\hline T. \\
\hline V \\
\hline
\end{tabular}}

FiO2, promedio (DE)

$\mathrm{n}=18$

Volumen corriente, promedio (DE)

Frecuencia respiratoria, promedio (DE)

PEEP, promedio (DE)

$70.83(22.24)$

Presión meseta, promedio (DE)

Distensibilidad, promedio (DE)

Tabla 3. Gasimetría arterial.

Variables

$\mathrm{pH}$, promedio (DE)

$\mathrm{n}=18$

$\mathrm{pCO} 2$, promedio (DE)

$7.27(0.22)$

pO2, promedio (DE)

$48.29(18.91)$

Saturación, promedio (DE

$68.76(17.28)$

PaFi, promedio (DE)

$88.1(12.02)$

Tabla 4. Puntaje severidad del SDRA.

Puntaje

$\mathrm{n}=18$

Índice oxigenación ajustado, promedio (DE)

$90.45(19.77)$

Puntaje Murray, promedio (DE)

$2.95(0.49)$

Puntaje APPS, promedio (DE)

$5.94(1.16)$

\section{Severidad según Clasificación de Berlin}

Leve, no. (\%)

Moderado, no. (\%)

$10(55.56)$

Severo, no. (\%)

$8(44.44)$

\section{Conclusiones}

Los puntajes de severidad APPS e índice de oxigenación ajustado pueden ser aproximaciones valiosas para predecir la mortalidad a mediano plazo. Deben realizarse estudios prospectivos, con representación adecuada de pacientes en todos los niveles de severidad, que permitan evaluar la validez externa de estas herramientas.

\section{Referencias}

1. Villar J, Ambrós A, Soler JA, Blanco J, Martín-rodríguez C, Fernández M, et al. Age, Pao2/Fio2, and Plateau Pressure Score: A Proposal for a Simple Outcome Score in Patients With the Acute Respiratory Distress Syndrome. Crit Care Med 2016; 44:1361-1369

2. Dechert RE, Park PK, et al. Evaluation of the oxygenation index in adult respiratory failure. I Trauma Acute Care Surg. 2014; 76(2): 46973. 